\title{
Publisher's Note: Nonparametric Bayesian inference of the microcanonical stochastic block model
} [Phys. Rev. E 95, 012317 (2017)]

Tiago P. Peixoto

(Received 19 January 2017; published 26 January 2017)

DOI: 10.1103/PhysRevE.95.019904

This paper was published online on 17 January 2017 with an error in the text of the abstract. The first line of the abstract should read as "A principled approach to characterize the hidden structure of networks is to formulate generative...." The paper has been corrected as of 20 January 2017. The text is correct in the printed version of the journal. 